\title{
Concentration- and Solvent-Dependent Photochemical Instability of 6,13-Bis(triisopropysilylethynyl)pentacene
}

\author{
Laila Abu-Sen, John J. Morrison, Andrew B. Horn,* and Stephen G. Yeates*
}

Solution processing of organic electronic materials is becoming commonplace for device fabrication since it provides a facile route to controlled, patterned thin films. Many of the candidate species however contain intense chromophores and a qualitylimiting factor in device fabrication is photochemical degradation in the solution phase. Pentacene for example has a very short half-life in solution under the influence of light; this behaviour can be attenuated markedly by derivatisation at the 6- and 13- positions. ${ }^{[1-3]}$ As a consequence, materials such as 6,13-bis(triisopropylsilylethynyl)pentacene (TIPS-pentacene) are becoming popular replacements for pentacene in electronic devices. ${ }^{[4,5]}$ However, these derivatives are themselves still prone to photochemical degradation during processing, which potentially perturbs both the optical absorption and electronic properties of films cast from solution. Upon UV irradiation $(\sim 300 \mathrm{~nm})$, TIPS-pentacene forms dimers by cycloaddition ${ }^{[6]}$ and at visible wavelengths is known to photodegrade in solution via a process involving dissolved oxygen. ${ }^{[7]}$ The predominant mechanism for this molecule involves activation of $\mathrm{O}_{2}$ molecules by the initially-formed excited state; this either generates singlet $\mathrm{O}_{2}$ species or a superoxide $\mathrm{O}_{2}^{-}$which then adds back across appropriate carbon centres in TIPS-pentacene to produce an endoperoxide (EPO), which is seen as the main product by NMR. ${ }^{[7]}$ Recent studies using chemically generated ${ }^{[8,9]}$ singlet $\mathrm{O}_{2}$ have further demonstrated that the superoxide route is the most favoured of the two for TIPS-pentacene, contributing around $90 \%$ to the overall rate of EPO formation. Electron transfer rates from excited-state pentacenes are also observed to be fast and solvent-dependent. ${ }^{[3]}$ Deactivation of the initial singlet state by mechanisms which can compete with $\mathrm{O}_{2}$ activation therefore serves to increase photostability. The main non-radiative mechanism for this is singlet fission ${ }^{[10,11]}$ via interaction with a second TIPS-pentacene molecule to produce a pair of triplets. This has been studied extensively in thin films of pentacene and its derivatives. ${ }^{[12-15]}$ Studies of the photochemistry of pentacene monomers and dimers in host crystals indicate that the singlet lifetime is much shorter in dimers than monomers, confirming the importance of pair-wise interactions. ${ }^{[16]}$ Luminescence

Dr. L. Abu-Sen, Dr. J. J. Morrison, Dr A. B. Horn,

Prof. S. G. Yeates

School of Chemistry

University of Manchester Oxford Road

Manchester, M13 9PL, UK

E-mail: Andrew.Horn@manchester.ac.uk;

stephen.yeates@manchester.ac.uk

This is an open access article under the terms of the Creative Commons Attribution License, which permits use, distribution and reproduction in any medium, provided the original work is properly cited.

DOI: 10.1002/adom.201400003 lifetimes are also strongly environment-dependent, ranging from around $7 \mathrm{~ns}$ in dilute solution ${ }^{[17]}$ to sub-ps in thin films. ${ }^{[15]}$ Direct observation of the kinetics of singlet fission of TIPSpentacene in anhydrous, $\mathrm{O}_{2}$-free $\mathrm{CHCl}_{3}$ solution over a range of concentrations reveals a singlet lifetime of $\sim 13 \mathrm{~ns},{ }^{[18]}$ whilst a high-concentration triplet yield of $200 \%$ and triplet formation at a rate approaching the diffusion limit indicates the efficiency of the process. Physical quenching must also be considered as a deactivation route in any condensed medium. Stability in solution is therefore controlled by the rates and quantum yields of these processes, many of which are not known. Recent reports of half-life increase by the attachment of stable radical species to pentacenes is further evidence of the importance of the initial singlet state lifetime. ${ }^{[19]}$

In this communication, a systematic study of the half-life of TIPS-pentacene in a variety of air-equilibrated common solvents (including toluene, tetrahydrofuran (THF), dichloromethane, chloroform, isopropanol (IPA), decane and dodecane) by UVvisible spectroscopy at concentrations ranging from $2.5 \times 10^{-6}$ $\mathrm{M}$ to $2 \times 10^{-2} \mathrm{M}$ is reported. Correlation of the variation of halflife for TIPS-pentacene photoxidation with TIPS-pentacene concentration, solubility parameters, dissolved $\mathrm{O}_{2}$ concentrations and intermolecular distances yields important new insight into the processes involved. In particular, a non-linear concentration dependence is observed which points to a further perturbation in solution due to the onset of aggregation.

UV-visible spectra for TIPS-pentacene in toluene over a range of concentrations from $270-800 \mathrm{~nm}$ in a $10 \mathrm{~mm}$ pathlength cuvette are characterized by three long-wavelength bands at 643, 592, and $549 \mathrm{~nm}$ (Figure 1a). These spectral features correspond to the known HOMO-LUMO gap with its associated vibrational structure. ${ }^{[20,21]}$ The $\lambda_{\max }$ values of the longest wavelength absorption do not appear to vary with concentration over the range shown but are observed to vary slightly with solvent (Supporting Information, S4.1). Upon photodegradation, these longer wavelength bands decrease with exposure whilst new features grow in below $450 \mathrm{~nm}$. Mass spectra and NMR spectra confirm that the main product is the 6,13-endoperoxide of TIPS-pentacene (Supporting Information, S1); this shows no features in the visible absorption spectra because the conjugated $\pi$-system in the product is too short (comprising 2 naphthalene rings). The new features observed below $450 \mathrm{~nm}$ are due to small quantities of the 5,14-endoperoxide (effectively a substituted anthracene. ${ }^{[6]}$ For each sample, plots of normalized $\mathrm{A} / \mathrm{A}_{0}$ at $643 \mathrm{~nm}$ versus time were made as shown in Figure 1b. These absorbance-time profiles were found to be reproducible to $+/-5 \%$, with all formulations showing retardation in rate beyond $t_{1 / 2}$. Comparable decays were also measured in THF, dichloromethane, IPA, chloroform, dodecane and decane over the same concentration range (Supporting Information, S2). 

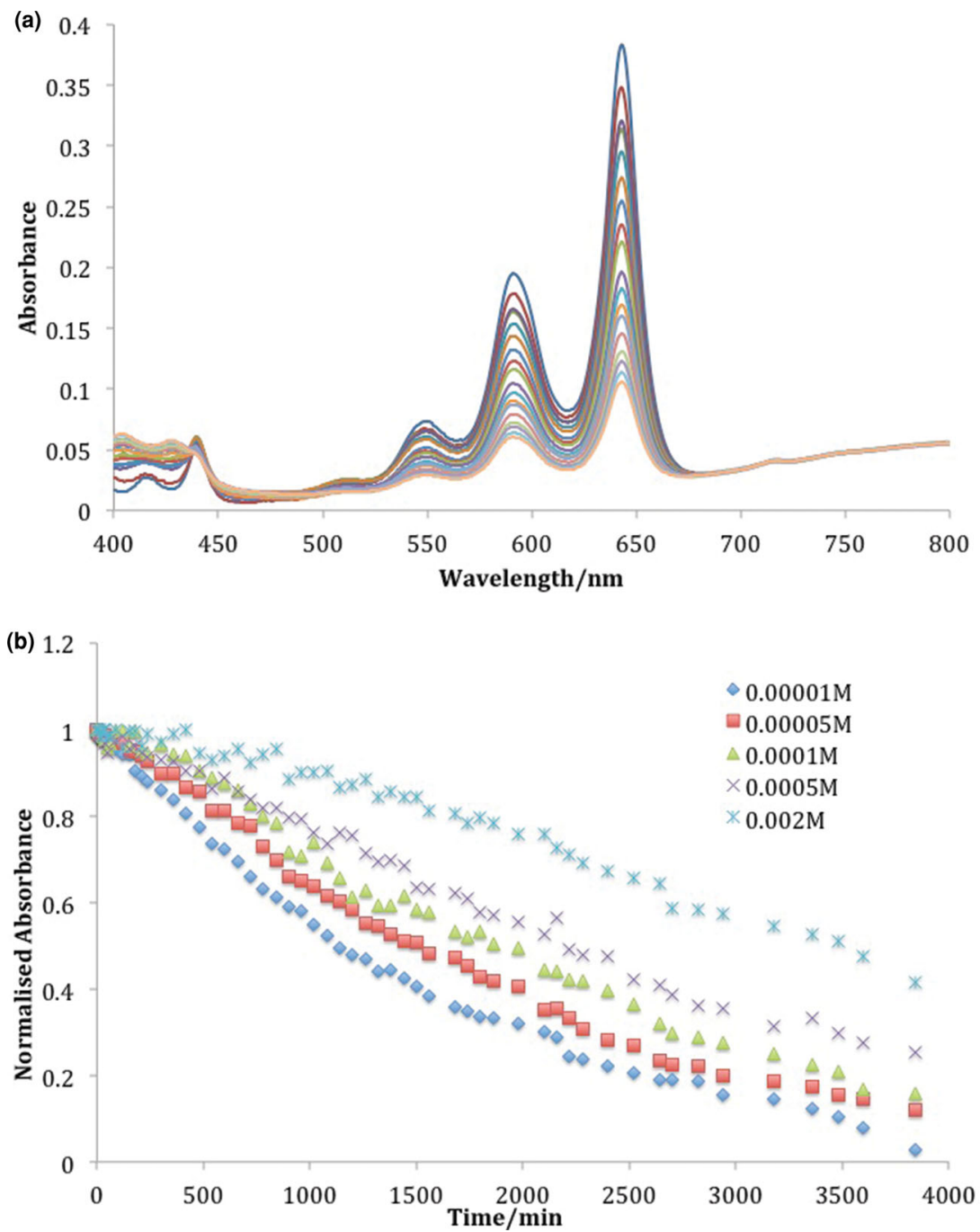

Figure 1. (a) UV-visible absorption spectra for the photodegradation of TIPS-pentacene in toluene at $1 \times 10^{-5} \mathrm{M}$ in a $10 \mathrm{~mm}$ pathlength. (b) Normalised absorbance/time profiles in toluene solution for a range of concentrations.

Figure 2 shows a summary plot of half-life data for TIPSpentacene photoxidation at concentrations ranging from $2.5 \times$ $10^{-6} \mathrm{M}$ to $2 \times 10^{-2} \mathrm{M}$. For all solvents, shortest half-lives are observed for lowest concentrations, with half-life increasing as concentration increases. For a number of solvents, the curves show inflection, typically between $1 \times 10^{-4}$ and $5 \times 10^{-4} \mathrm{M}$. This is particularly pronounced in the case of the chlorinated solvents and toluene.

The singlet fission process requires the proximity of a second TIPS-pentacene molecule, which implies that the efficiency of singlet fission should scale with nearest-neighbour distance in pure solvent, which in turn is a function of solution concentration. An estimate of the nearest neighbour distance as a function of TIPS-pentacene concentration can be obtained from cuberoot of the concentration (see Supporting Information, S3.1). Figure 3 shows a plot of the half-life versus mean intermolecular distance for the data presented in Figure 2. The variation of lifetime with distance can be seen to be approximately linear up to concentrations around $5 \times 10^{-4} \mathrm{M}$ (corresponding to a nearest neighbour distance of $\left.1.5 \times 10^{-8} \mathrm{~m}\right)$. Below this concentration, the kinetics of the photodegradation process are effectively first order, as shown in Figure 4 which is a plot of $\ln (\mathrm{A} /$ $A_{0}$ ) versus time. From these observations, it is clear that the increase in half-life is (in part) due to an increased likelihood of a second TIPS-pentacene molecules being sufficiently close to participate in triplet formation (and thereby deactivate the singlet state in preference to energy/electron transfer to $\mathrm{O}_{2}$ ).

Insight into the role of solvent in determining the half-life is forthcoming from a consideration of two factors: the 'goodness' of the solvent and the concentration of potential quenching species. The former can be expressed via Hansen solubility parameters (HSPs), which take into account the relative strengths of the dispersion $\left(d_{\mathrm{d}}\right)$, polar $\left(d_{\mathrm{p}}\right)$ and hydrogen bonding $\left(d_{\mathrm{h}}\right)$ forces between solvent and solute molecules (Supporting Information, S3). ${ }^{[22]}$ These parameters are used to define the centre of a solubility sphere for a given solute (here defined by TIPS-pentacene) and an interaction radius, $R_{\mathrm{a}}$, for any solvent:

$$
R_{a}^{2}=4\left(d_{d 1}-d_{d 2}\right)^{2}+\left(d_{p 1}-d_{p 2}\right)^{2}+\left(d_{h 1}-d_{h 2}\right)^{2}
$$

where subscripts are 1 for solute (TIPS-pentacene) and 2 for the solvent. Good solvents will have a small $R_{\mathrm{a}}$ (i.e. the solubility parameters are closely matched); typically the solute will be highly soluble in such solvents. Progressively poorer solvents have higher values (and declining solubility). There is a positive, albeit poor, correlation between half-life data for TIPS-pentacene at $1 \times 10^{-5} \mathrm{M}$ with $R_{\mathrm{a}}$ determined across a range of solvents, as shown in Figure S4.2, such that to a first order when $R_{\mathrm{a}}$ is small, half-lives are comparably low and when $R_{\mathrm{a}}$ is large half-lives are comparably large. Such a correlation (poor solvent $=$ large half-life) can be interpreted on the basis of simple nonideal solution thermodynamic arguments, i.e. as evidence that poor solvent drives TIPS-pentacene molecules together with a concomitant increase in the efficiency of singlet fission.

A second process which can be invoked to explain the reduction in the lifetime of the singlet state is quenching, for which dissolved oxygen is a prime candidate. Once again, a positive correlation exists between the concentration of dissolved oxygen $^{[23-25]}$ (expressed as mole-fraction $\mathrm{O}_{2}$ at saturation) and half-life (shown in Figure S4.3 for $1 \times 10^{-5} \mathrm{M}$ ), although again the correlation is not high. A clearer picture of the combination of these two processes can be seen from a plot of the product of the HSP and saturation oxygen solubility versus the half-life, as shown in Figure 5. Here, the correlation is much improved. A similarly high correlation is also obtained at $1 \times 10^{-4} \mathrm{M}$ but not at $1 \times 10^{-3} \mathrm{M}$ (see Supporting Information, S4). The reason 


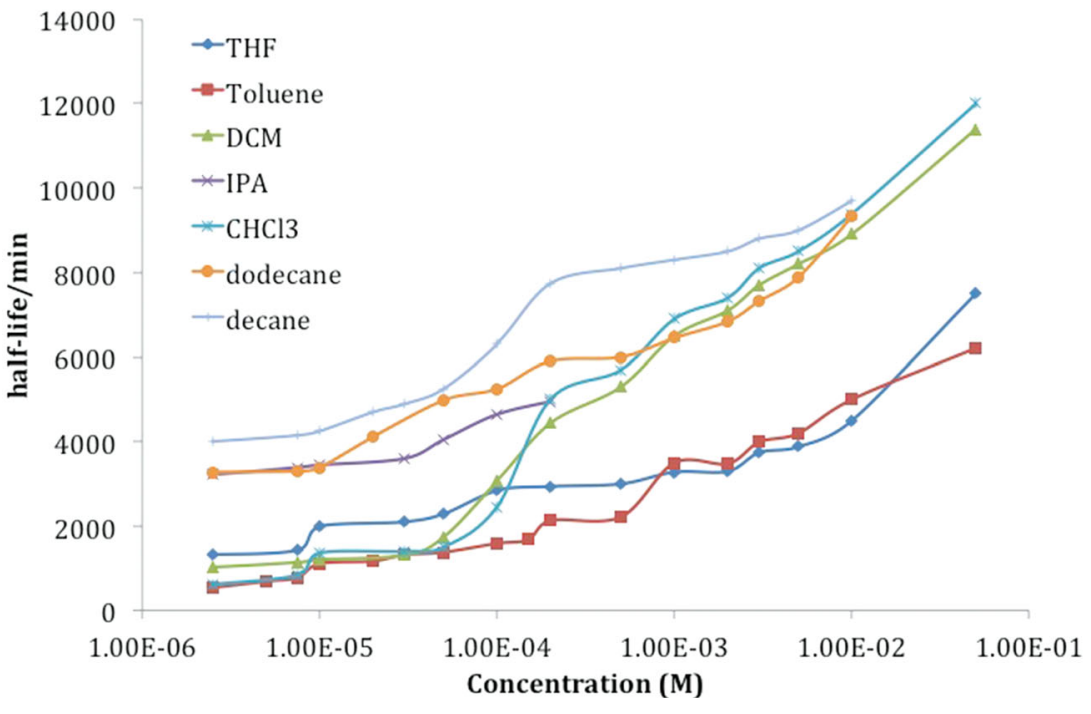

Figure 2. Variation of half-life as a function of concentration for TIPS-pentacene in a variety of solvents between $2.5 \times 10^{-6}$ to $5.0 \times 10^{-2} \mathrm{M}$. Note that the data range is limited by solubility in IPA, decane and dodecane (supporting information).

for this cut-off point is discussed below. It seems clear however that, at low concentration, the initial singlet state is deactivated by both intermolecular processes between TIPS-pentacene molecules and by quenching by dissolved oxygen.

Above concentrations of around $2 \times 10^{-4} \mathrm{M}$, the half-life is observed to increase non-linearly up to the solubility limit and the kinetics of photoxidation are no longer first order (e.g. the $2 \times 10^{-3} \mathrm{M}$ trace in Figure $\left.1(\mathrm{~b})\right)$. Given the known tendency of acenes to aggregate in solution, ${ }^{[26]}$ it seems likely that this behaviour reflects the onset of substantial aggregate formation. Direct evidence for aggregate absorption is not observable in the UV-visible spectra however since it is not possible to record spectra at concentrations above $2 \times 10^{-5} \mathrm{M}$ without saturation. Aggregation would have two principal effects upon photodegradation: first, since proximity to another TIPS-pentacene molecule serves to deactivate the initial excited singlet, molecules associated with aggregates (either on the surface or within the bulk) would therefore be less susceptible to oxidation; second, any molecule within an aggregate is physically separated from $\mathrm{O}_{2}$ in solution and would therefore be incapable of $\mathrm{O}_{2}$ activation. The mode of aggregation is also likely to play a role in determining the concentration-dependence of stability. There are two simple treatments of aggregation that can be considered here: isodesmic, and cooperative. ${ }^{[27-29]}$ In the former, aggregation occurs by equilibrium between free monomer and a series of successively higher $\mathrm{N}$-mers (dimer, trimer, teramer etc.) with a single equilibrium constant for the addition of a further monomer to each N-mer. In this case, the number of molecules which are aggregated (i.e. from dimers upwards) increases with concentration according to a $2.0 \times 10^{-2} \mathrm{M}$. predictable model (with a dependence upon the choice of $\mathrm{K}$, which is not known a priori but requires the data to be fitted). If deactivation is linked to isodesmic aggregation, then the half-life should therefore scale linearly with the proportion of non-aggregated molecules (if an appropriate value of $\mathrm{K}$ is used). However, there is no value of $\mathrm{K}$ that allows the data to be fitted across the entire concentration range for any solvent (see Supporting Information, S3.2). Furthermore, the linearity of the fit to nearest neighbour distance (which would be perturbed by concentration-dependent aggregation) and first-order kinetics suggests that the degree of aggregation is minimal at low concentrations. The second model which has been shown to be applicable to the aggregation of some acenes is that of cooperative aggregation, differing from the isodesmic model in that the equilibrium constant $\mathrm{K}^{\prime}$ for the formation of a dimer is different from that for subsequent aggregate growth (addition of monomer to N-mers of trimer and above). In this case, the proportion of aggregated molecules shows an onset threshold concentration above which aggregation occurs. ${ }^{[29]}$ The onset of aggregation should therefore be expected to be accompanied by an increase in half-life. Since this is what is observed here (particularly in the case of the chlorinated solvents, which show a substantial increase in half-life at $1 \times 10^{-4} \mathrm{M}$ ), it is concluded that the perturbation which increases the half-life beyond that controlled by solvent quality and quenching (both by nearest-neighbours and $\mathrm{O}_{2}$ ) is therefore cooperative aggregation with an onset around 2-5 $\times$ $10^{-4} \mathrm{M}$, with some slight variation between solvents. Indeed, the kinetics of aggregation should depend upon the solvent, with good solvent restricting aggregation (and possibly having an influence on the mode of aggregation). The onset concentration for putative aggregation $\left(\sim 5 \times 10^{-4} \mathrm{M}\right)$ in the data presented

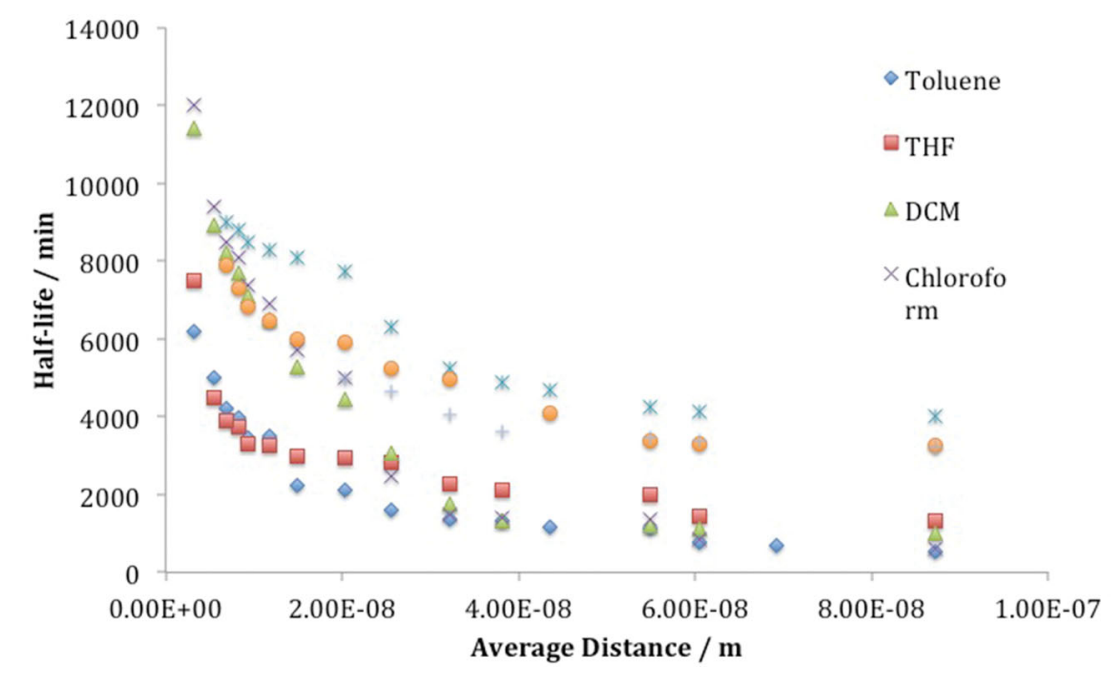

Figure 3. Variation of half-life versus average intermolecular distance between $2.5 \times 10^{-6}$ to 


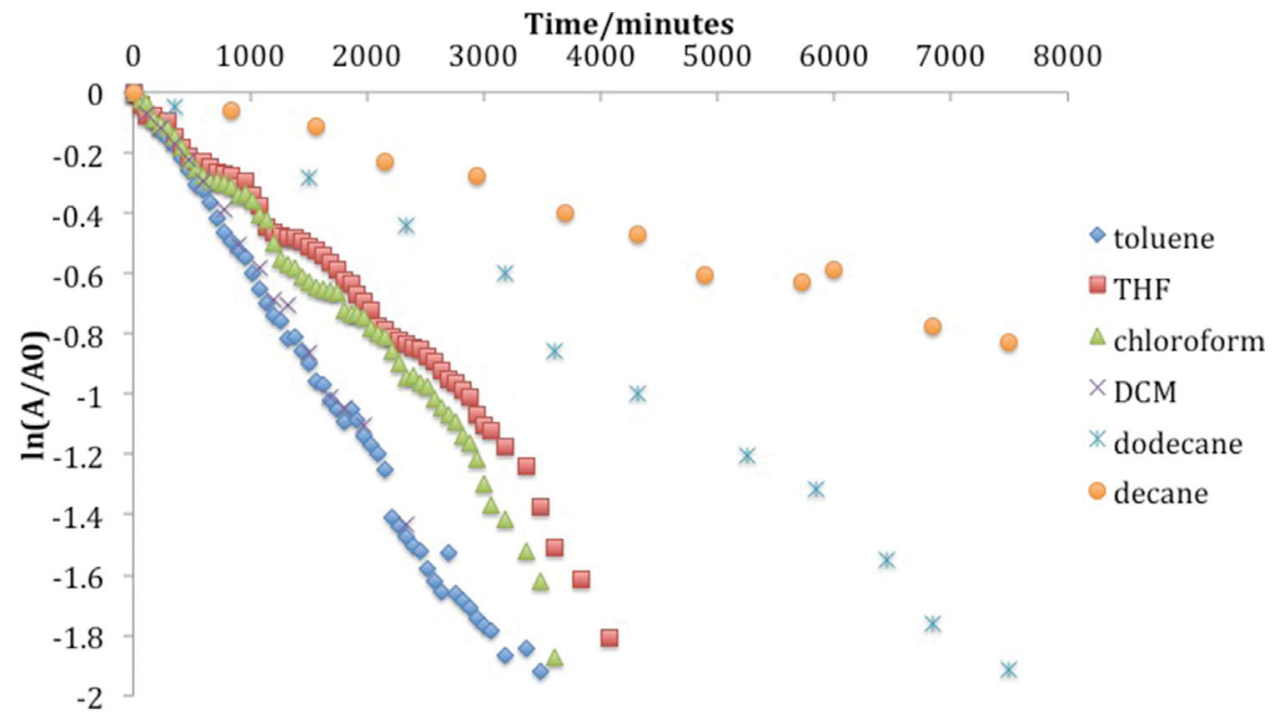

Figure 4. (a) Plot of $\ln \left(A / A_{0}\right)$ versus time for TIPS-pentacene in a variety of solvents at a concentration of $1 \times 10^{-5} \mathrm{M}$.

here lies just below the threshold for efficient singlet fission reported by Walker et al. $\left(>1 \times 10^{-3} \mathrm{M}\right) ;{ }^{[18]}$ furthermore, the triplet yield versus concentration reported in the above study is visually comparable to the degree of aggregation expected for a cooperative process (cf. Figure $1 \mathrm{C}$ in ref. [29] and Figure 3 in ref. [18]). It should also be noted that, although Walker et al. discount aggregate formation in their experiments on the basis of DOSY-NMR spectroscopy, there is evidence in the literature that this technique is unlikely to be particularly sensitive to small $\pi$-stacked aggregates since the hydrodynamic volume of small aggregates (dimers, trimers etc.) is unlikely to be significantly different from monomers. ${ }^{[30,31]}$ Further experiments are needed to clarify this point.

In summary, these measurements clearly demonstrate the effects of solvent quality, oxygen solubility, near-neighbour interactions and aggregation on the photostability of acenes in solution. At low concentration, photoexcited TIPS-pentacene

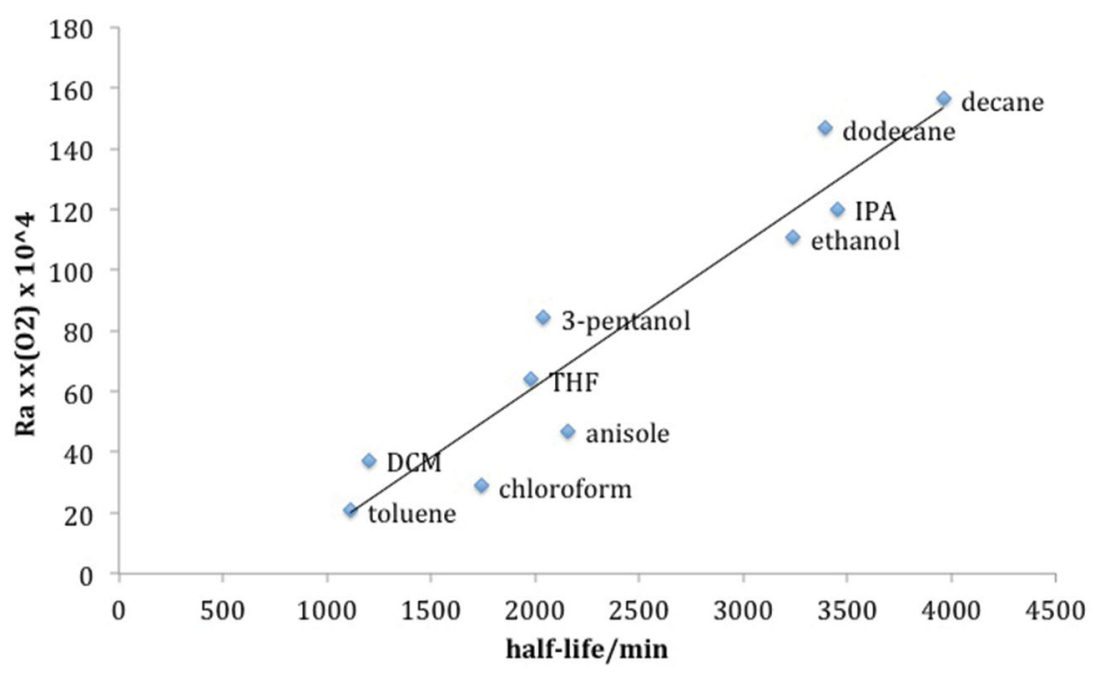

Figure 5. (a) Plots of half-life versus the product of the Hansen solubility parameter $R_{\mathrm{a}}$ and the mole-fraction of dissolved $\mathrm{O}_{2}$ at a concentration of $1 \times 10^{-5} \mathrm{M}$ TIPS-pentacene. molecules have a lower probability of deactivation and tend to preferentially undergo electron-transfer to $\mathrm{O}_{2}$, leading to short life-times with respect to endoperoxide formation. This effect is mitigated by poor solvent and by high oxygen solubility, which serve to deactivate the initial excited state by singlet fission and quenching respectively. At higher concentrations, photostability is increased by de-excitation as above and also by singlet fission due to the formation of aggregates (which are both incapable of reacting with oxygen and also effectively quench the initial singlet state of TIPS-pentacene, as observed in condensed thin films). These observations have ramifications for device fabrication. If the concentration is too low, the rate of photodegradation is significant and it is likely that decomposition products with unfavourable optical or electronic properties will be incorporated into devices. At higher concentrations, aggregates are present in significant numbers and, although this increases photostability, the incorporation of pre-formed aggregates into films may have implications for the structure (and therefore electronic and/or optical properties) of the resulting devices. However, these effects might be mitigated or controlled by careful manipulation of the solute/solvent blend: such an approach requires a much more sophisticated understanding of the intermolecular interactions in ternary blends. These observations may also go some way to help understand the effect that subtle changes in solvent character in both binary and ternary mixtures can have upon the thin film crystallisation of substituted acenes from solution and their resultant thin film transistor performance. ${ }^{[32-34]}$ Further work to examine the photochemistry and photophysics of substituted pentacenes in solution is therefore needed to understand these processes at a molecular level and the interdependency with resultant device performance. 


\section{Experimental Section}

TIPS-pentacene was synthesized using the Swager modification of Anthony's synthesis. ${ }^{[35]}$ Purified TIPS-pentacene was characterized by a combination of ${ }^{1} \mathrm{H}$ and ${ }^{13} \mathrm{C}$ NMR spectroscopies, UV-visible spectroscopy, high-resolution mass spectrometry (HR-MS) and cyclic voltammetry (CV). Kinetic measurements were performed on purified TIPS-pentacene dissolved in solvent, which had previously been exposed to air for 24 hours by loosening the cap to allow for free exchange of air, over a range of concentrations and formulations. UV-visible spectra were obtained on a Nicolet Evolution 300 spectrometer using $1 \mathrm{~cm}$ quartz cells. The cells were protected from light until each experiment began, at which point an initial spectrum was recorded to define $A_{0}$. The cells were placed on a laboratory bench under conventional $60 \mathrm{~W}$, SP35 fluorescent lighting (General Electric, $2850 \mathrm{~lm}$ ). The solutions were repeatedly scanned at prescribed intervals until less than $5 \%$ of the starting acene remained. For concentrations below $1 \times 10^{-5} \mathrm{M}$, UV-visible spectra were recorded without further dilution. For solutions at higher concentrations, samples were degraded under the same conditions and further diluted to $1 \times 10^{-5} \mathrm{M}$ immediately prior to the recording of spectra.

\section{Supporting Information}

Supporting Information is available from the Wiley Online Library or from the author.

\section{Acknowledgements}

JM was supported by funding from the Engineering and Physical Sciences Research Council (UK) via a Flagship Grant (FS/01/01/10) from the Innovative Electronic Manufacturing Research Centre, Loughborough. LA was supported by the Libyan Ministry of Education and partial sponsorship by Merck Chemicals Limited.

The copyright line for this article was changed on November 18, 2014.

Received: January 6, 2014

Revised: March 10, 2014

Published online: April 3, 2014

[1] I. Kaur, W. Jia, R. P. Kopreski, S. Selvarasah, M. R. Dokmeci, C. Pramanik, N. E. McGruer, G. P. Miller, J. Am. Chem. Soc. 2008, 130, 16274-16286.

[2] A. Maliakal, K. Raghavachari, H. Katz, E. Chandross, T. Siegrist, Chem. Mater. 2004, 16, 4980-4986.

[3] B. H. Northrop, K. N. Houk, A. Maliakal, Photochem. Photobiol. Sci. 2008, 7, 1463

[4] J.-H. Kwon, S.-I. Shin, J. Choi, M.-H. Chung, H. Kang, B.-K. Ju, Solid State Electronics 2009, 53, 266-270.

[5] G. R. Llorente, M. B. Dufourg-Madec, D. J. Crouch, R. G. Pritchard, S. Ogier, S. G. Yeates, Chem. Commun. 2009, 3059-3061.

[6] P. Coppo, S. G. Yeates, Adv. Mater. 2005, 17, 3001-3005.
[7] W. Fudickar, T. Linker, J. Am. Chem. Soc. 2012, 134, 15071-15082.

[8] D. Zehm, W. Fudickar, M. Hans, U. Schilde, A. Kelling, T. Linker, Chem.-Eur. J. 2008, 14, 11429-11441.

[9] D. Zehm, W. Fudickar, T. Linker, Angew. Chem. Int. Ed. 2007, 46, 7689-7692.

[10] M. B. Smith, J. Michl, Chem. Rev 2010, 110, 6891-6936.

[11] M. B. Smith, J. Michl, Annu. Rev. Phys. Chem. 2013, 64, 361-386.

[12] C. Ramanan, A. L. Smeigh, J. E. Anthony, T. J. Marks, M. R. Wasielewski, J. Am. Chem. Soc. 2011, 134, 386-397.

[13] A. Rao, M. Wilson, S. Albert-Seifried, R. Di Pietro, R. Friend, Phys. Rev. B 2011, 84, 195411.

[14] M. W. B. Wilson, A. Rao, J. Clark, R. S. S. Kumar, D. Brida, G. Cerullo, R. H. Friend, J. Am. Chem. Soc. 2011, 133, 11830-11833.

[15] P. M. Zimmerman, F. Bell, D. Casanova, M. Head-Gordon, J. Am. Chem. Soc. 2011, 133, 19944-19952.

[16] F. G. Patterson, H. Lee, W. L. Wilson, M. D. Fayer, Chem. Phys. $1984,84,51-60$.

[17] N. Nijegorodov, V. Ramachandran, D. P. Winkoun, Spectrochim. Acta A 1997, 53, 1813-1824.

[18] B. J. Walker, A. J. Musser, D. Beljonne, R. H. Friend, Nature Chem. 2013, 5, 1019-1024.

[19] Y. Kawanaka, A. Shimizu, T. Shinada, R. Tanaka, Y. Teki, Angew. Chem. Int. Ed. Engl. 2013, 52, 6643-6647.

[20] J. Aragó, P. M. Viruela, E. Ortí, R. Malavé Osuna, V. Hernández, J. T. López Navarrete, C. R. Swartz, J. E. Anthony, Theoret. Chem. Acc. 2010, 128, 521-530.

[21] Z. Zhu, S. Wu, Y. Zhang, Russian J. Phys. Chem. A 2008, 82, 2293-2298.

[22] C. M. Hansen, Hansen Solubility Parameters: A User's Handbook, 2nd ed., CRC Press, Boca Raton 2007.

[23] R. Battino, T. R. Rettich, T. Tominaga, J. Phys. Chem. Ref. Data 1983, 12, 163-178.

[24] K. Fischer, J. Chem Thermodynam. 2001, 33, 1285-1308.

[25] K. Shirono, T. Morimatsu, F. Takemura, J. Chem. Engineering Data 2008, 53, 1867-1871.

[26] M.-B. Madec, S. Butterworth, P. Taboada, R. Heenan, M. Geoghegan, S. G. Yeates, Soft Matter 2011, 7, 7065.

[27] Z. Chen, A. Lohr, C. R. Saha-Möller, F. Würthner, Chem. Soc. Rev. 2009, 38, 564.

[28] D. Zhao, J. S. Moore, Org. Biomol. Chem. 2003, 1, 3471.

[29] M. M. J. Smulders, M. M. L. Nieuwenhuizen, T. F. A. De Greef, P. Van Der Schoot, A. P. H. J. Schenning, E. W. Meijer, Chem.-Eur. J. 2010, 16, 362-367.

[30] Z. Chen, V. Stepanenko, V. Dehm, P. Prins, L. D. A. Siebbeles, J. Seibt, P. Marquetand, V. Engel, F. Würthner, Chem.-Eur. J. 2007, 13, 436-449.

[31] E. M. García-Frutos, G. Hennrich, E. Gutierrez, A. Monge, B. Gómez-Lor, J. Org. Chem. 2010, 75, 1070-1076.

[32] R. S. Clough, D. H. Redinger, J. C. Novack, Patent No. WO 2009/151978 A1, 2009.

[33] X. Li, B. K. C. Kjellander, J. E. Anthony, C. W. M. Bastiaansen, D. J. Broer, G. H. Gelinck, Adv. Funct. Mater. 2009, 19, 3610-3617.

[34] M.-B. Madec, P. J. Smith, A. Malandraki, N. Wang, J. G. Korvink, S. G. Yeates, J. Mater. Chem. 2010, 20, 9155.

[35] Y. Kim, J. E. Whitten, T. M. Swager, J. Am. Chem. Soc. 2005, 127, 12122-12130. 